\title{
7 Der Uber-Moment in der Krankenversicherung
}

\author{
Evelyn Kade-Lamprecht
}

\section{Die Erosion hat begonnen - Der digitale Flaschenhals wird enger}

Der Wettlauf der Krankenkassen um die Gunst der Kunden, die für sich selbst und für ihre Angehörigen permanent auf der Suche nach der persönlich besten Gesundheitsversorgung sind, ist im vollen Gange. Um Kunden zu begeistern, ist der Zusatzbeitrag in der Krankenversicherung ein ebenso ungeliebtes Thema, wie die Buchpreisbindung im Buchhandel, wie die GEMA-Gebühren im Musikgeschäft oder wie die $\mathrm{CO}$-Bilanz im Transportgewerbe.

Für die Krankenversicherungen wird der digitale Flaschenhals enger. Zuhauf schwappen disruptive Impulse und technologische Trends aus völlig anderen Branchen in den Gesundheitsmarkt, die die Versicherten wie selbstverständlich auch von ihrer Krankenkasse erwarten.

Während die Autowelt bereits fertige Konzepte dafür in der Schublade hat, dass es das Auto bald nur noch als Add on-Service „dazugeben“ wird, quälen sich die Digitalisierungsteams der Krankenkassen mit den Datenschutzregularien der Aufsichtsbehörden herum. So tüfteln die großen Automobilkonzerne bereits heute daran, wie das Auto der Zukunft durch Sensortechnik und Wearables zu einem digitalen Gesundheitsbegleiter werden kann. Fitness-Assistenten, die während der Fahrt die Vitalwerte messen und ggf. an die Notrufzentrale weiterleiten, sind längst keine ScienceFiction mehr.

Ebenso wie in der Automobilindustrie läuft auch in der Krankenversicherung die Bestandsgarantie alter, bekannter Marktplayer aus. So hat zum Beispiel das amerikanische Start-up Uber eine ganze Branche wachgerüttelt, indem das Prinzip 
der „Share Economy“ in das Taxigewerbe Einzug hielt. Ein weiteres Beispiel: Als Digital Hero der Automobilbranche hat Tesla die Rituale einer mehr als hundertjährigen Industrie gesprengt und treibt diese mit den Mitteln der Tech-Branche nunmehr vor sich her. Tesla hat das Auto zum Softwareprodukt gemacht, das wie ein Smartphone über Updates stets auf dem neuesten Stand gehalten wird (Freitag u. Rest 2017).

Während die Krankenversicherungsbranche lange in vergleichsweise ruhigen Fahrwassern agierte, hat nun die Erosion begonnen. Im Mausklicktempo wachsen neue Wettbewerber heran. In Amerika ist die Idee eines internetbasierten Krankenversicherers mit Oscare längst Realität. Und in Deutschland sitzt der digitale Krankenversicherer Ottonova in den Startlöchern, um den Markt der privaten Krankenversicherer durcheinanderzuwirbeln. Völlig neue Marktplayer schieben sich zwischen die alteingesessenen Krankenversicherer und ihre Kunden, darunter junge innovative Start-ups, deren Gesundheitsprodukte auf reges Interesse bei der onlineaffinen Klientel stoßen.

Werden deutsche Krankenversicherer bald ihren eigenen Uber-Moment erleben oder werden sie es besser als das Taxigewerbe machen, das vom disruptiven Geschäftsmodell „Uber“ überrollt wurde?

\section{Science-Fiction bald Realität?}

In ihren innersten Vorstelllungen und Erwartungen an die Krankenkasse der Zukunft sind die Kunden den Krankenversicherungen bereits weit voraus (TCP 2016). Sowohl Kranke als auch Gesunde zeigen ein hohes Interesse daran, in Sachen Gesundheit proaktiv von der Krankenkasse beraten zu werden. Die Patientendaten dazu zu nutzen, ihre Gesundheit zu fördern und zu schützen, erscheint den Versicherten logisch und sinnvoll. „Wenn die Kasse alle meine Daten schon hat, soll sie damit auch was machen, [...] mich darauf hinweisen, welche Behandlungsmöglichkeiten sie hat" (TCP 2016).

Ohne intelligente Technologie ist eine proaktive Versorgungssteuerung allerdings unmöglich. Hier sind die Krankenkassen im Zugzwang, denn für die Versicherten stehen in diesem Zusammenhang der Nutzen einer digitalen Patientenakte sowie einer personalisierten Webseite außer Zweifel. Bislang ist den Kassen eine proaktive Versorgungssteuerung aus Datenschutzgründen nicht erlaubt. Die Versicherten befinden sich allerdings bereits auf der Überholspur. Ihre unorthodoxe Denke zeichnet für scheinbar Unmögliches ein klares Bild, wie sich die Datenschutzbedenken technologisch ausräumen ließen.

Einfach umsetzbar ist der Kundenvorschlag, die Kassen könnten vorab eine individuelle Einwilligung der Versicherten einholen. Welche Daten die Patienten der Kasse für eine proaktive Versorgungssteuerung freigeben, wollen die Kunden dabei selbst bestimmen. „Wie bei PayPal oder anderen Portalen kann man das Angebot individuell zuschneiden [...] Speicherung von Anamnese, Röntgenbild, Notfalldaten, Sterbeabsicherung, Patientenverfügung" (TCP 2016).

Sehr viel weiter gedacht und fast wie Science-Fiction anmutend ist der Denkanstoß der Kunden, einen implantierbaren telemetrischer Gesundheits-Chip zu nutzen: „Mir wär lieber ein Chip unter die Haut. Das zahlen wir ja sowieso. Kann man ja in 
den Science-Fiction-Filmen schon überall sehen“(TCP 2016). So neu und überraschend diese Einblicke in die echte Kundendenke für eine Krankenversicherung auch erscheinen mögen, für den Versicherten sind das längst keine Gimmicks, sondern Gesundheitsdienstleistungen mit klarer Verhaltensrelevanz.

\section{Like or Dislike - Die Kunden sind wählerisch}

In der Krankenkasse der Zukunft werden Apps, Chats, Online-Coachings, intelligente Selfservice-Tools und digitale Assistenten selbstverständlich sein. Ungern „kaufen“ und nutzen die Kunden aber „die Katze im Sack“, sondern sie wägen stattdessen den persönlichen Nutzen der digitalen Krankenkassen-Angebote für ihre konkrete Gesundheitssituation sorgfältig ab (s. Abb. 1).

Bei aller Digitalisierung ist sowohl bei den gesunden als auch bei den kranken Versicherten der Wunsch allgegenwärtig, alternativ durch einen persönlichen Ansprechpartner begleitet zu werden, der im Bedarfsfall individuell berät. Während die meisten Krankenkassen in der Realität im Telefon-, E-Mail- und Chatverkehr auf anonyme Hotlines und Callcenter setzen, zeichnen die Versicherten für ihre Krankenversicherung von morgen ein anderes Zukunftsbild: Eine menschliche Gesundheitsfürsorgerin, die in ihrer starken Gemeinschaft mit effizienter Kommunikation aktiv persönlich berät und sich für ihre mündigen Mitglieder einsetzt.

Ohne Investitionen in modernste Technik und Technologie bleibt den Kassen die Tür in die Zukunft allerdings verschlossen. Von voll digitalisierten Geschäftsmodellen ist die Krankenversicherungsbranche in Deutschland bislang noch weit entfernt. Mit ein paar Klicks und Wischern über den Touchscreen lästige Standard-KV-Angelegenheiten erledigen, bei Bedarf den Arzt per Videosprechstunde konsultieren oder den Gesundheitszustand und die Vitaldaten mittels künstlicher Intelligenz überwachen

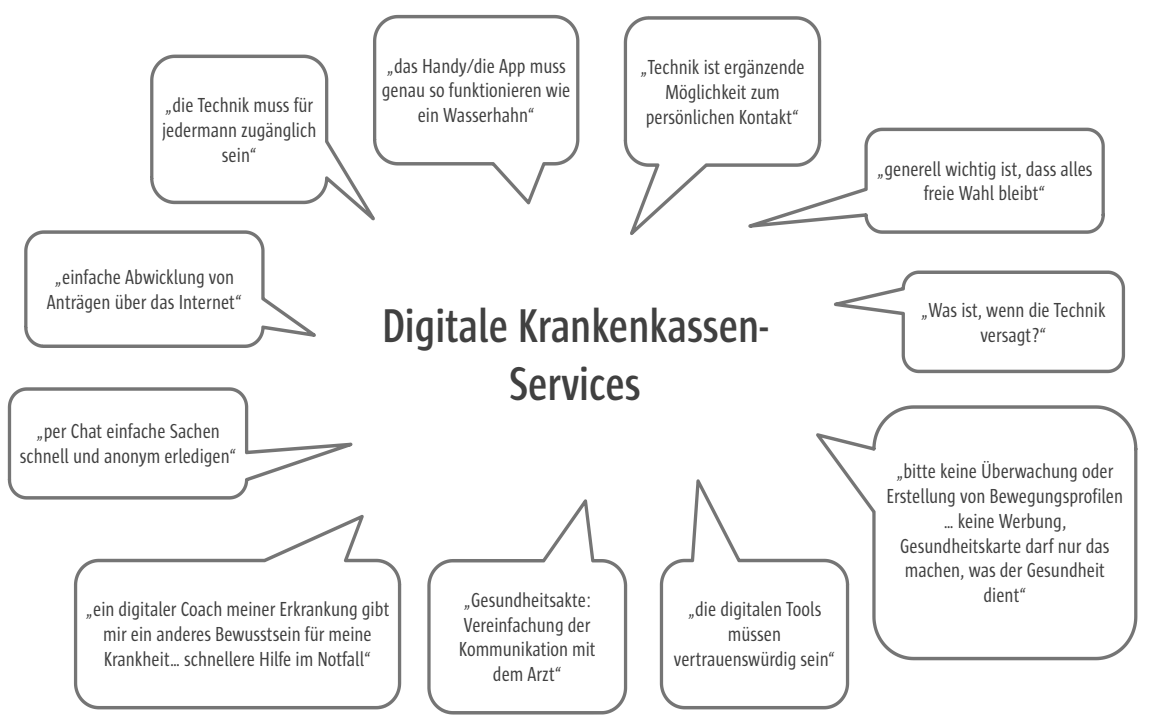

Abb. 1 Like or Dislike: Der Kunde entscheidet selbst auf seiner digitalen Reise (TCP 2016) 
lassen - smarte Assistenten ermöglichen das Begleiten des Versicherten in den verschiedenen Gesundheits- und Krankheitssituationen.

Allerdings möchte der Kunde an jeder Stelle seiner digitalen Reise selbstbestimmt entscheiden, welche digitalen Services, Therapieangebote, Kommunikationskanäle er für sich persönlich nutzen will. Daher ist für die Kasse der Zukunft die digitale Transformation nur zu schaffen, wenn es gelingt, die eigene Organisation und alle Prozesse um den Kunden herum zu strukturieren und dabei den Versicherten in den Mittelpunkt zu stellen.

\section{Start-ups sind outgesourcte Innovation}

„Wenn Apple eine Krankenkasse aufmachen würde, würde ich es machen, weil ich denke, dass ich individueller betreut würde“ (TCP 2016). Die Kunden kreieren eine völlig neue Messlatte für das Ökosystem „Krankenkasse“, das sich im Digitalisierungszeitalter plötzlich mit branchenfremden Wettbewerbern, wie z.B. dem Technologiekonzern Apple vergleichen lassen muss.

Um echte Innovationen in den hochregulierten Gesundheitsmarkt zu bringen, darf ein Ausharren in den gelernten analogen Denkrastern einer Krankenversicherung keine Option mehr sein. Der digitale Tsunami ist unaufhaltsam unterwegs. Da er sich nicht stoppen lässt, tun Krankenversicherungen gut daran, ihn mitzugestalten:

- Statt langwierigen Nachdenkens ist „Querdenken“ gefragt.

- Statt Perfektion und immer mehr Features sollte es um „Einfachheit“, „Praktikabilität“ und „Gesundheitsnutzen“ für die Versicherten gehen.

- Statt „Abwarten“ bringt „Machen“ den nötigen Marktvorsprung.

Junge innovative E-Health-Start-ups werkeln mit Hochdruck daran, ihre Geschäftsmodelle für das Andocken an die Krankenversicherung salonfähig zu machen. Datenschutz und Medizinproduktezertifizierung der smarten Gesundheitsangebote sind mittlerweile Standard und daher für die meisten Start-ups längst keine KO-Kriterien für den Schritt in den ersten Gesundheitsmarkt mehr. Die innovativen Start-ups und E-Health-Technologieunternehmen führen den Kassen immer wieder vor Augen, wie gefangen diese in ihren eigenen Strukturen sind.

Ähnlich wie bei Uber, Apple, Google, Amazon und Facebook werden die Start-ups und Technologiepioniere neue Schnittstellen kreieren, die in naher Zukunft über den Erfolg des Geschäftsmodells Krankenversicherung mitentscheiden können. Dabei wird die Vernetzung aller Marktteilnehmer verpflichtend.

So wie die Fahrzeughersteller das Airbnb-Konzept auf die Straße gebracht haben, wird der Uber-Effekt die Krankenversicherungsbranche massiv durcheinanderwirbeln, wenn sie sich der Kooperation mit den Start-ups und Technologiepionieren verwehren.

Dabei ist „Zeit“ die neue Währung. Zum Vergleich: Bei Uber oder Tesla geht es um Tage, Stunden und Minuten. Wer einmal hinten liegt, hat gleich mehrere Jahre Rückstand. In der Krankenversicherung überdauern die Entscheidungsprozesse zu neuen digitalen Lösungen nicht selten die Lebenszeit eines Start-ups. 


\section{Fazit}

Das still im Hintergrund laufende Betriebssystem „Krankenkasse“ hat ausgedient. Die meisten Krankenkassen haben erkannt, dass sie gegen digitale Disruptoren nicht immun sind. Nun ist Handeln, Experimentieren und Ausprobieren gefragt. Nutzen Sie den Innovationsvorsprung der Digital Health-Start-ups, indem Sie Ihr Geschäftsmodell für Innovationen öffnen. Schaffen Sie frühzeitig die richtigen technologischen Schnittstellen, indem Sie die Start-ups als outgesourcte Forschungs- und Entwicklungsabteilung in Ihre Prozesse integrieren.

\section{Literatur}

Freitag M, Rest I (2017) Manager Magazin 2/2017: 22-27

TCP Terra Consulting Partners GmbH (2016) Die Krankenkasse der Zukunft 